\title{
Medical education at the University of São Paulo Medical School
}

\author{
Joaquim Edson Vieira,' Luiz Fernando Ferraz da Silva," Edmund Chada Baracat"' \\ 'Faculdade de Medicina da Universidade de São Paulo, Department of Surgery, Discipline of Anesthesiology, São Paulo/SP, Brazil. "Faculdade de Medicina \\ da Universidade de São Paulo, Department of Pathology, São Paulo/SP, Brazil. "'IFaculdade de Medicina da Universidade de São Paulo, Department of \\ Gynecology and Obstetrics, Discipline of Gynecology, São Paulo/SP, Brazil.
}

E-mail: edmund.baracat@hc.fm.usp.br

The Faculdade de Medicina da Universidade de São Paulo (FMUSP) is beginning a new undergraduate medical curriculum in 2015. This model reduces the time allotted to lectures and increases the time devoted to tutored discussions and to clinical reasoning, without neglecting the need to acquire information and scientific foundations that guide the practice of medicine, ensuring that the curriculum is always grounded in the best evidence. Anchored in research precepts from the field of medical education, resulting from numerous large meetings with teachers and students, and supported by international experts, the new FMUSP curriculum accounts for several important dilemmas in medical education.

Included among these dilemmas are the continuous and cumulative acquisition of new scientific information; new approaches to health care and the treatment of acute and chronic diseases; and recognition of social, economic and cultural determinants of health for both individuals and populations.

The following examples may illustrate the most common dilemmas faced by medical educators for the layperson. In particular, the advent of magnetic resonance imaging did not replace computed tomography, which in turn did not replace ultrasonography, which did not replace X-ray exams, and so forth. It is thus necessary to know the principles of all of these techniques. As another example, this time using the medical literature [http://www.ncbi.nlm.nih.gov/pubmed], between the 1970s and 2010, the time necessary to study all of the texts considered as well-established content (articles and reviews in medicine) increased from $20 \%$ of the time dedicated to a medical course, or 10,000 hours in six years, to sevenfold. That is, if medical training depended only on theoretical content, the addition of new information would result in the completion of such training requiring approximately 40 years (assuming no new developments over those 40 years).

In practice, graduating with a medical degree in 6 years (which is traditional in Brazil) is no longer considered the

Copyright @ 2015 CLINICS - This is an Open Access article distributed under the terms of the Creative Commons Attribution Non-Commercial License (http:// creativecommons.org/licenses/by-nc/3.0/) which permits unrestricted non-commercial use, distribution, and reproduction in any medium, provided the original work is properly cited. end of education. This is clear when we observe that doctors never exhaust the need to update their knowledge with both "sensu lato" post-graduate courses and other forms of continued education. However, there is a need for systematic education during undergraduate education, focusing on basic information and nuclear or fundamental medical knowledge and centering the process on the student, who must finish medical school in six years with the ability to continue learning (learning to learn). Additionally, complementary to the addition of new knowledge in the field of medicine, the theme of "social determinants of health" [http://www.ssrn.com/en/] has become more frequent in the medical literature since the 1990s and has significantly highlighted the need for new generations of health professionals to study and perform research.

Building a medical curriculum is not a simple or quick task. This process requires beginning with a strong and dynamic basis, followed by continuous evaluation for longterm improvement. Along these lines, the FMUSP constantly discusses its curriculum, which is quite healthy. However, the institution recently decided to reinforce its national leadership with a new curricular model. This model, presented here, was created based on interviews with all coordinators of the FMUSP Disciplines and Clerkship, which evolved into working groups, or task forces that worked to integrate the thematic content for the basic and clinical sciences. Specifically, between 2012 and 2014, international experts in medical education from the US, Canada and Portugal visited the FMUSP and, through a collaborative and ongoing dialogue, including both students and faculty, created the new curriculum. The new curriculum therefore arose from the curricula of many other medical schools, with important additional contributions from professors in other units of the Universidade de São Paulo, scholars from the field of medical education, and medical experts as well as researchers, all of whom are dedicated and committed to the training of FMUSP students. The curriculum is thus the result of an institutional effort that involved both the FMUSP and the University via three institutes (Biomedicine/ICB, Chemistry/IQ, and Biosciences/IB) that are involved in medical education.

The term used for the current stage of development of this new curriculum has been "capillarity" (a good choice, considering its medical relevance) because medical education now affects everyone's (troubled) daily life via FMUSP teachers and students as well as hospital doctors, whose roles as professors of medicine are essential and recognized. 
No unanimity is expected from smart institutions; disagreement is an important force driving further development and improvement. The most prepared institutions show resilience (mainly before destabilizing events), adapting and changing for the better on the verge of new developments, which are always arising.

We at the USP Medical School are all excited to welcome the new and to support what has been consolidated, as well as being hopeful, committed and engaged. Nevertheless, no one is puzzled by or indifferent to the challenges. This is a good sign for all; with this new curriculum, we want to be even closer to society to offer the highest levels of health care and relevant scientific production and to participate in social development with ethics, humanism and transparency. In particular, the Universidade de São Paulo hopes to maintain its leadership and to ensure the education of the best doctors, scientists and citizens to serve society. 